Article

\title{
Effective Generalized Partial Response Target and Serial Detector for Two-Dimensional Bit-Patterned Media Recording Channel Including Track Mis-Registration
}

\author{
Thien An Nguyen $(\mathbb{D}$ and Jaejin Lee *(D) \\ Department of Information Communication Convergence Technology, Soongsil University, Seoul 06978, Korea; \\ anthienng1995@soongsil.ac.kr \\ * Correspondence: zlee@ssu.ac.kr; Tel.: +82-2-820-0901
}

Received: 23 July 2020; Accepted: 14 August 2020; Published: 19 August 2020

check for updates

\begin{abstract}
With the development of 5G technology, programs are gradually moving to cloud services. This leads to an increasing demand for storage. In the field of high-density data storage, bit-pattern media recording (BPMR) is considered a promising approach, as it can expand the data density to $4 \mathrm{~Tb} / \mathrm{in}^{2}$. However, in high-density BPMR, bits or magnetic islands are very close to each other, leading to significant intertrack interference (ITI) from the cross-track direction and intersymbol interference (ISI) from the down-track direction. To minimize two-dimensional interference, including ITI and ISI, the serial detector method has been highly effective. However, in this method, the signal at the output of the first decoder is still a hard output. Therefore, we suggest methods to convert the output of the first detector into a soft output. Additionally, we have developed a new form of generalized partial response target to overcome the track mis-registration. The results show that our proposed methods apparently improve bit error rate performance.
\end{abstract}

Keywords: information storage; bit-patterned media recording; intersymbol interference; partial response maximum likelihood; soft-output viterbi algorithm

\section{Introduction}

In the field of data storage, conventional magnetic recording systems have an area density limit of $\sim 1 \mathrm{~Tb} / \mathrm{in}^{2}$ because of the superparamagnetic effect [1]. Therefore, bit-patterned media recording (BPMR) has been proposed as a promising candidate to solve this problem. In BPMR, one bit is stored in a single-domain island surrounded by a nonmagnetic region, and the areal density (AD) can reach up to $4 \mathrm{~Tb} / \mathrm{in}^{2}$ [2]. Moreover, BPMR technology can decrease the nonlinear transition shift, offer easier tracking, and exhibit good thermal stability [2,3]. For increasing the storage capacity, the distance between magnetic islands must be closer, which produces intersymbol interference (ISI) and intertrack interference (ITI) from the down-track (horizontal) and cross-track (vertical) directions, respectively. These types of interference are referred to as two-dimensional (2D) interference in BPMR systems.

Many methods are used to reduce 2D interference. We can use modulation methods like those of Nguyen [4], with an error-correcting 5/6 code to avoid fatal interference as much as possible and gain error correction; of Buajong [5], with a combination of a rate-3/4 modulation code and ITI subtraction to reduce ITI; and of Kanjanakunchorn [6], with a rate-5/6 constructive ITI code to handle ITI. We can use detection in combination with a partial response (PR) target and an equalizer. To reduce ITI, Nabavi [7] proposed a modified trellis for BPMR. In [8], Nabavi suggested a 2D equalizer with a PR target and optimized equalizer coefficients to eliminate ISI and ITI. Based on Nabavi $[7,8]$, a hybrid 2D equalizer was proposed by Wang [9] to improve channel estimation when ITI is known. Additionally, 
to mitigate 2D interference, Kim [10] proposed a 2D soft-output Viterbi algorithm (2D SOVA), which is also known as a parallel detection model, for holographic data storage, then developed an iterative 2D SOVA for bit-patterned media [11]. Jeong, based on the parallel detection model, proposed a multipath ISI structure that fits the staggered BPMR structure [12]. In addition to the idea of parallel detection, recently the idea of serial detection appeared [13]. In serial detection, the 2D interference is separated into two serial interference components. This helps reduce the complexity of 2D Viterbi detection of the BPMR channel with 2D interference. However, in serial detection, the inner detection still has an output that is referred to as a hard output. This is a disadvantage of serial detection compared to parallel detection, which has a soft output in inner detection.

In this study, we propose two methods to create a soft output for the inner detector. The first method is to use an equalizer to create a soft output. The second method is based on the Viterbi algorithm combined with channel interference to create a soft output. This method is highly effective, because it can reduce errors compared to a six-level signal and preserve the interference information of the signal. Moreover, when the channel experiences track mis-registration (TMR), which degrades system performance $[14,15]$, the previous PR target is no longer effective in overcoming TMR. Therefore, we have proposed a new form of PR target for the 2D BPMR channel with TMR. Finally, we investigated the ability of serial detection in the presence of media noise.

The rest of this paper is organized as follows. In Section 2, we explain our algorithm and show how we design the soft output for serial detection. Section 3 briefly presents the BPMR channel model and illustrates the simulation results. Finally, the conclusion is drawn in Section 4.

\section{New PR Target and Soft Output for Serial Detection}

\subsection{New PR Target for $T M R$}

To implement serial detection, the PR target with polynomial $G$ has the following matrix form [13].

$$
\mathbf{G}=\left[\begin{array}{ccc}
r p & p & r p \\
r & 1 & r \\
r p & p & r p
\end{array}\right],
$$

where $r$ and $p$ are the interference coefficients from horizontal and vertical directions, respectively. This form has a coefficient at four corners, which is the product of horizontal and vertical coefficients, and the ITI coefficients are symmetric. Consequently, the equalizer output can be analyzed as given below:

$$
=a[j, k] *\left[\begin{array}{ccc}
r p & p & r p \\
r & 1 & r \\
r p & p & r p
\end{array}\right]=a[j, k] *\left[\begin{array}{c}
p \\
1 \\
p
\end{array}\right] *\left[\begin{array}{ccc}
r & 1 & r
\end{array}\right],
$$

where $*$ is the convolution operator.

With the above analysis, the 2D interference of the channel includes two serial one-dimensional (1D) interference components. In other words, the original signal is distorted by vertical interference then horizontal interference. Therefore, the detection composed of a 1D horizontal detector in series with a 1D vertical detector, as shown in Figure 1.

Since the channel is no longer symmetric in the vertical direction when the system has TMR, the upper interference coefficient $\left(g_{-1.0}\right)$ and the lower interference coefficient $\left(g_{1,0}\right)$ are estimated with different values. Therefore, a new form of PR target such as is required.

$$
\mathbf{G}=\left[\begin{array}{ccc}
r p_{1} & p_{1} & r p_{1} \\
r & 1 & r \\
r p_{2} & p_{2} & r p_{2}
\end{array}\right]
$$


With this asymmetric form, a signal $z[j, k]$ like (2) is given as and applied to the serial detector, as shown in Figure 1.

$$
\begin{aligned}
& z[j, k] \approx a[j, k] * G \\
& =a[j, k] *\left[\begin{array}{ccc}
r p_{1} & p_{1} & r p_{1} \\
r & 1 & r \\
r p_{2} & p_{2} & r p_{2}
\end{array}\right]=a[j, k] *\left[\begin{array}{c}
p_{1} \\
1 \\
p_{2}
\end{array}\right] *\left[\begin{array}{ccc}
r & 1 & r
\end{array}\right]
\end{aligned}
$$

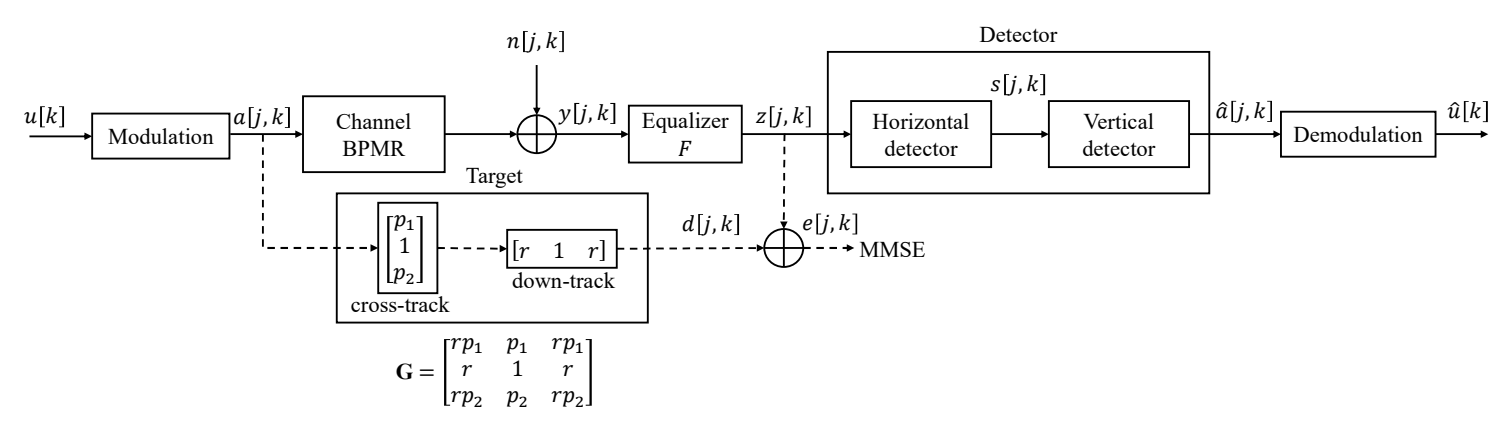

Figure 1. Serial detection scheme.

\subsection{Soft Output Using an Equalizer}

In the serial detector for the PR target without TMR as shown in Figure 1, the first (inner) detector converts the real-valued equalizer output signal $z[j, k]$ into the six-level signal $s[j, k]$ (they are $-2 p-1,-1$, $2 p-1,-2 p+1,1$, and $2 p+1$.) and then the second (outer) detector converts the signal into the original signal a[j,k], which is the 2-level signal [13] since the signal $s[j, k]$ is a six-level output. This loses the interference information and reduces system performance. To solve this problem, we propose two methods. First, we design an equalizer for replacing the inner detector, because the equalizer can estimate a signal similar to the output of the inner detector and create a soft output, which helps preserve the interference information. The system with the equalizer is presented in Figure 2.

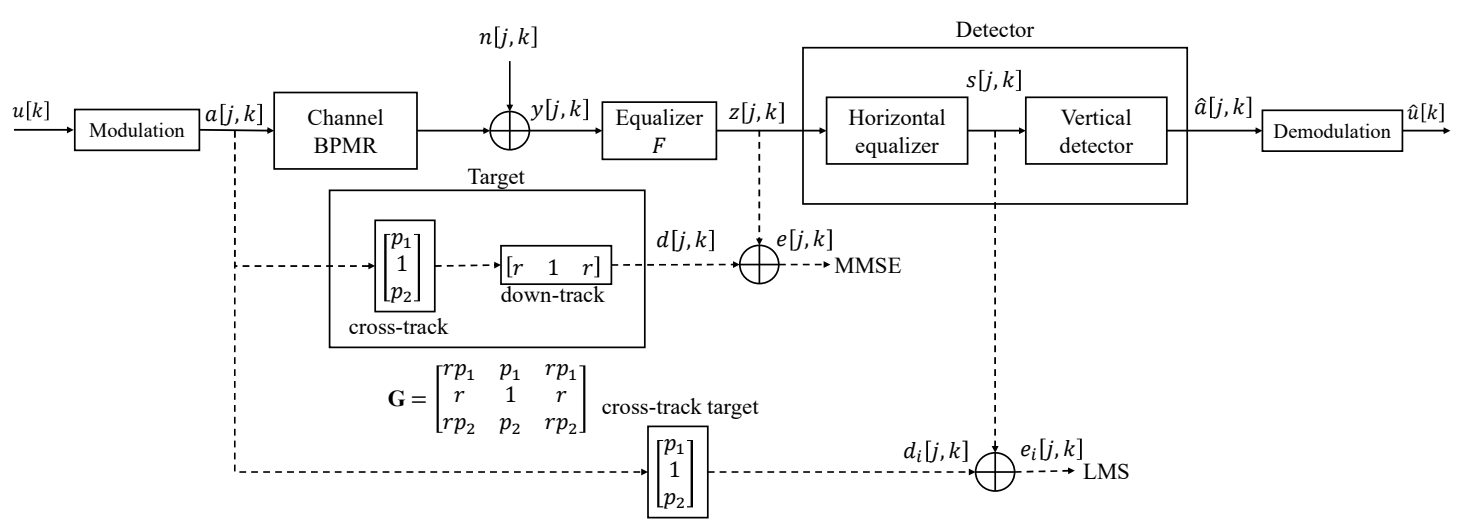

Figure 2. System with a horizontal equalizer replacing the horizontal (inner) detector.

In the model, the coefficient of target $\mathrm{G}$ is estimated by using a minimum mean square error (MMSE) method. After finding the coefficients of target $G$, we have the horizontal (down-track) and vertical (cross-track) target coefficients, respectively. Based on the coefficients of cross-track, the horizontal equalizer can be designed for replacing the inner detector. The coefficients of the horizontal equalizer, which replaces the inner detector, are estimated by using the least mean square (LMS) algorithm. 


\subsection{Soft Output Utilizing a Horizontal Detector and Interference}

With the above method, the equalizer substituting for the inner detector produces a soft output for the outer detector. However, this method produces signals that have more errors than a six-level signal. To remove this error, we have to use the Viterbi algorithm at the inner detector to restore a six-level signal like a conventional serial detector. We then design a feedback unit to ascertain the interference information and add the interference information with the six-level signal. The model of the inner detector with soft output is presented in Figure 3.

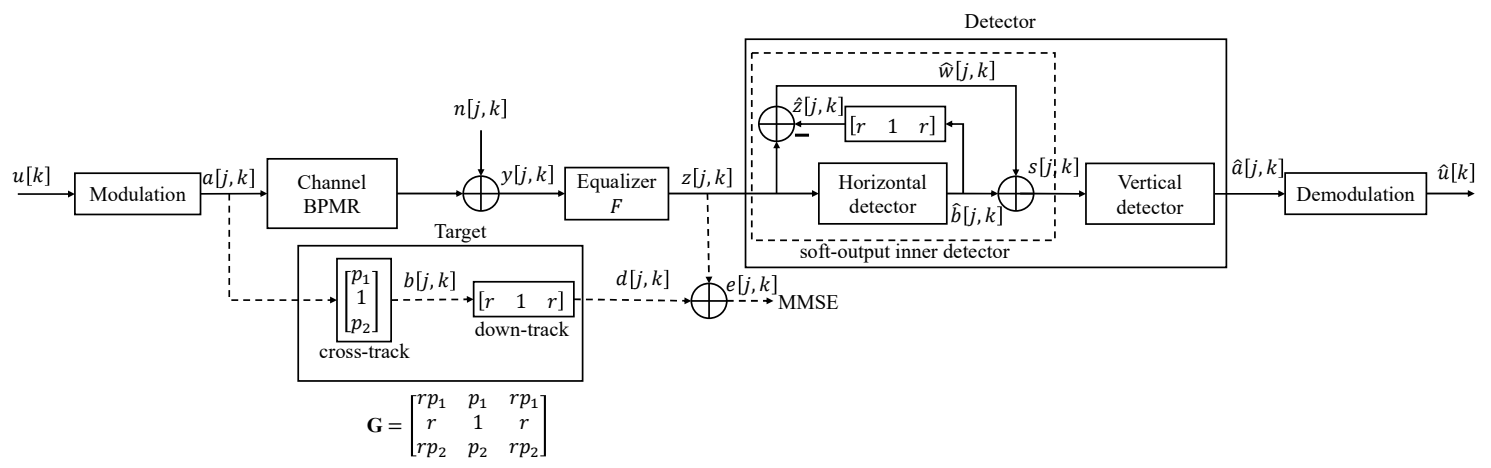

Figure 3. System with a soft-output horizontal (inner) detector.

In Figure 3, the signal $z[j, k]$, which is approximately a 2D convolution of the target and the original data $a[j, k]$, is presented as follows:

$$
\begin{aligned}
& z[j, k] \approx a[j, k] * G+w[j, k] \\
& =a[j, k] *\left[\begin{array}{c}
p_{1} \\
1 \\
p_{2}
\end{array}\right] *\left[\begin{array}{lll}
r & 1 & r
\end{array}\right]+w[j, k],
\end{aligned}
$$

where $w[j, k]$ is an additive distortion component, i.e., colored noise plus residual interference. For ease of signal analysis, we set $b[j, k]=a[j, k] *\left[\begin{array}{c}p_{1} \\ 1 \\ p_{2}\end{array}\right]$. We can represent (5) as follows

$$
z[j, k] \approx b[j, k] *\left[\begin{array}{lll}
r & 1 & r
\end{array}\right]+w[j, k] .
$$

The signal $z[j, k]$ is passed through the inner detector. In an ideal condition, the inner detector converts the signal $z[j, k]$ into the signal $b[j, k] \in\{-2 p-1,-1,2 p-1,-2 p+1,1,2 p+1\}$, in which the horizontal interference has been eliminated. However, in fact, because of the noise signal $w[j, k]$, it only restores the approximation of $b[j, k]$. We refer to that signal as $\hat{b}[j, k]=b[j, k]+e_{\mathrm{b}}[j, k]$. When continuing to use the $\hat{b}[j, k]$ signal for the outer detection, the performance is degraded. To solve this issue, we estimate the noise $w[j, k]$. With $\hat{b}[j, k]$, we predict the noise signal $w[j, k]$ and set it to $\hat{w}[j, k]$ according to the following equations:

$$
\begin{gathered}
\hat{z}[j, k]=\hat{b}[j, k] *\left[\begin{array}{ccc}
r & 1 & r
\end{array}\right]=b[j, k] *\left[\begin{array}{ccc}
r & 1 & r
\end{array}\right]+e_{b}[j, k] *\left[\begin{array}{lll}
r & 1 & r
\end{array}\right], \\
\hat{w}[j, k]=z[j, k]-\hat{z}[j, k]=w[j, k]-e_{b}[j, k] *\left[\begin{array}{lll}
r & 1 & r
\end{array}\right] .
\end{gathered}
$$

The signal $\hat{w}[j, k]$ is the noise information that is added to the output signal of the inner detector. Thus, the final output of the inner detector is calculated as follows:

$$
s[j, k]=\hat{b}[j, k]+\hat{w}[j, k]=b[j, k]+e_{b}[j, k]-e_{b}[j, k] *\left[\begin{array}{lll}
r & 1 & r
\end{array}\right]+w[j, k] .
$$


Meanwhile, the $r$ coefficient is usually quite small. So $e_{\mathrm{b}}[j, k]^{*}[r 1 r]$ is close to $e_{\mathrm{b}}$

$$
e_{b}[j, k] \approx e_{b}[j, k] *\left[\begin{array}{ccc}
r & 1 & r
\end{array}\right] .
$$

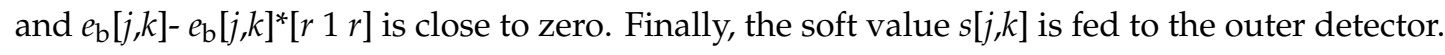

$$
s[j, k] \approx b[j, k]+w[j, k] .
$$

\section{Simulation Results}

\subsection{BPMR Channel Model}

With the original data $u[k] e\{0,1\}$, it is modulated into the $2 \mathrm{D}$ data array $a[j, k]$ e $\{-1,1\}$. The input data $a[j, k]$ are taken into the BPMR channel, in which the signals are interfered by ISI and ITI. At the output of the channel, additive white Gaussian noise is added to present the noise model. In this research, we apply a 2D Gaussian function to represent the 2D island response of the BPMR channel as follows [12]:

$$
P(x, z)=A \exp \left(-\frac{1}{2 c^{2}}\left[\left(\frac{x+\Delta_{x}}{P W_{x}}\right)^{2}+\left(\frac{z+\Delta_{z}}{P W_{z}}\right)^{2}\right]\right),
$$

where $x$ and $z$ are the down- and cross-track directions, respectively; $\Delta_{x}$ and $\Delta_{z}$ are the down- and cross-track bit location fluctuations, respectively; $c$ is $1 / 2.3548$, which represents the relationship between the standard deviation of a Gaussian function and $\mathrm{PW}_{50}$, which is a parameter of the pulse width at half of the peak amplitude; and $\mathrm{PW}_{x}$ and $\mathrm{PW}_{z}$ are the $\mathrm{PW}_{50}$ components of the down- and cross-track pulses, respectively.

The BPMR channel pulse response is expressed as

$$
h[j, k]=P\left(j T_{x}, k T_{z}-\Delta_{o f f}\right),
$$

where $j$ and $k$ are the discrete indices in the down- and cross-track directions, respectively; $T_{x}$ and $T_{z}$ are the bit period and track pitch, respectively, and $\Delta_{\text {off }}$ is the read-head offsets for the cross-track. TMR is defined as the ratio between the head offset size and the magnetic-island period, as follows:

$$
\operatorname{TMR}(\%)=\frac{\Delta_{o f f}}{T_{z}} .
$$

The readback signal $y[j, k]$ for BPMR is given by

$$
y[j, k]=a[j, k] * h[j, k]+n[j, k],
$$

where $a[j, k], h[j, k]$, and $n[j, k]$ are the 2D discrete input data, 2D channel response, and electronic noise modeled as additive white Gaussian noise (AWGN) with variance $\sigma^{2}$ and zero mean, respectively.

\subsection{Simulation Results}

In this experiment, the first method is shown in Figure 2, which uses an equalizer instead of the inner detector. The channel output $y[j, k]$ was inputted to $2 \mathrm{D}$ equalizer. This equalizer has a size of $5 \times 5$. At the same time, the coefficients of the equalizer and the GPR target were determined by calculating the error values $e[j, k]$ and minimizing this error with the MMSE algorithm [13]. Then, the output of the equalizer $z[j, k]$ was passed to the horizontal equalizer, which had a size of $5 \times 5$, and the coefficients were updated by finding the error values $e_{i}[j, k]$ and using the LMS algorithm to reduce ISI. Finally, the outputs of the horizontal equalizer, $s[j, k]$, were transferred to the vertical detector to reduce ITI and restore the original input data $\hat{a}[j, k]$. The channel signal-to-noise ratio (SNR) is defined as $10 \log _{10}\left(1 / \sigma^{2}\right)$, where $\sigma^{2}$ is AWGN power. To simulate the second method, we built a scheme as shown in Figure 3. The system with the six-level output signal for serial detection was built in the same 
manner as Figure 1. In our experiment, we refer to the system in Figure 3 as soft-output horizontal (inner) detection, the system in Figure 2 as horizontal (inner) equalizer, and system in Figure 1 as conventional serial detection.

First, we compare the bit error rate (BER) performance of three systems as shown in Figure 4. We simulated 10 pages with a size of $1200 \times 1200$ bits and an AD of $3 \mathrm{~Tb} / \mathrm{in}^{2}\left(T_{x}=T_{z}=14.5 \mathrm{~nm}\right)$ [16]. To simplify the problem, the coefficients of the channel without TMR effect and media noise used in the simulation were given as

$$
\mathbf{H}=\left[\begin{array}{ccc}
0.0824 & 0.3876 & 0.0824 \\
0.2125 & 1 & 0.2125 \\
0.0824 & 0.3876 & 0.0824
\end{array}\right]
$$

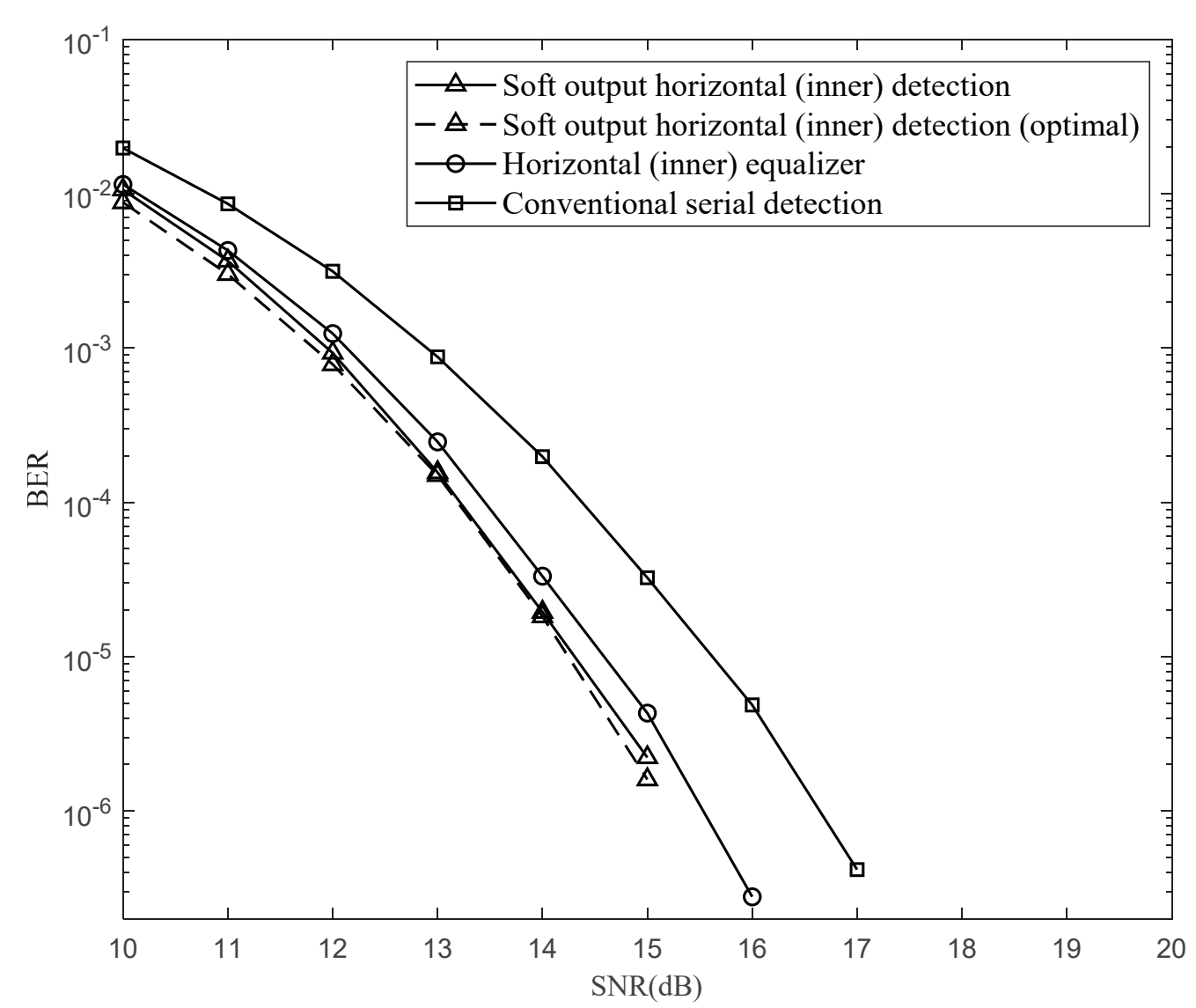

Figure 4. Bit error rate (BER) performance of the proposed models without track mis-registration (TMR).

At $\mathrm{SNR}=20 \mathrm{~dB}$, the PR target was calculated as

$$
\mathbf{G}=\left[\begin{array}{ccc}
0.0503 & 0.4348 & 0.0503 \\
0.1158 & 1 & 0.1158 \\
0.0503 & 0.4348 & 0.0503
\end{array}\right]
$$

The results showed that the system with soft output of the inner detector achieves better BER performance than the system with hard output of the inner detector. At a BER of $10^{-6}$, the gain of the soft output horizontal detector is $\sim 1.6 \mathrm{~dB}$ higher than that of the conventional serial detection and $0.5 \mathrm{~dB}$ higher than that of the horizontal equalizer. Because soft output preserves the interference information, the outer detector decides more accurately. In Figure 3, the soft-output inner detector has two functions. One is to reduce errors compared to a six-level signal, which improves the accuracy 
of the inner detector. The other is the preservation of interference information. The resulting noise information is then provided to the outer detector, which is the second detector in the serial detector, thus improving the performance of the entire serial detector. For GPR target to reach the optimal coefficient, which is the same as the channel coefficients, we choose the matrix $\mathbf{G}=\mathbf{H}$. The result of this case is represented by the dashed line in Figure 4, and the proposed scheme is close to the optimal performance.

In the next simulation, we include the effect of TMR and the channel matrix $\mathbf{H}$ is changed according to the level of TMR (\%). We show the examples of 10 and 20\% TMRs. The system is simulated when $\mathrm{AD}$ is $3 \mathrm{~Tb} / \mathrm{in}^{2}$. The BER performance of this simulation with $10 \%$ and $20 \%$ TMR is shown in Figures 5 and 6 , respectively. The coefficients of channels with $10 \%$ and $20 \%$ TMR were shown as

$$
\mathbf{H}_{10 \%}=\left[\begin{array}{lll}
0.0675 & 0.3176 & 0.0675 \\
0.2105 & 0.9906 & 0.2105 \\
0.0986 & 0.4641 & 0.0986
\end{array}\right]
$$

and

$$
\mathbf{H}_{20 \%}=\left[\begin{array}{lll}
0.0543 & 0.2554 & 0.0543 \\
0.2046 & 0.9628 & 0.2046 \\
0.1159 & 0.5452 & 0.1159
\end{array}\right],
$$

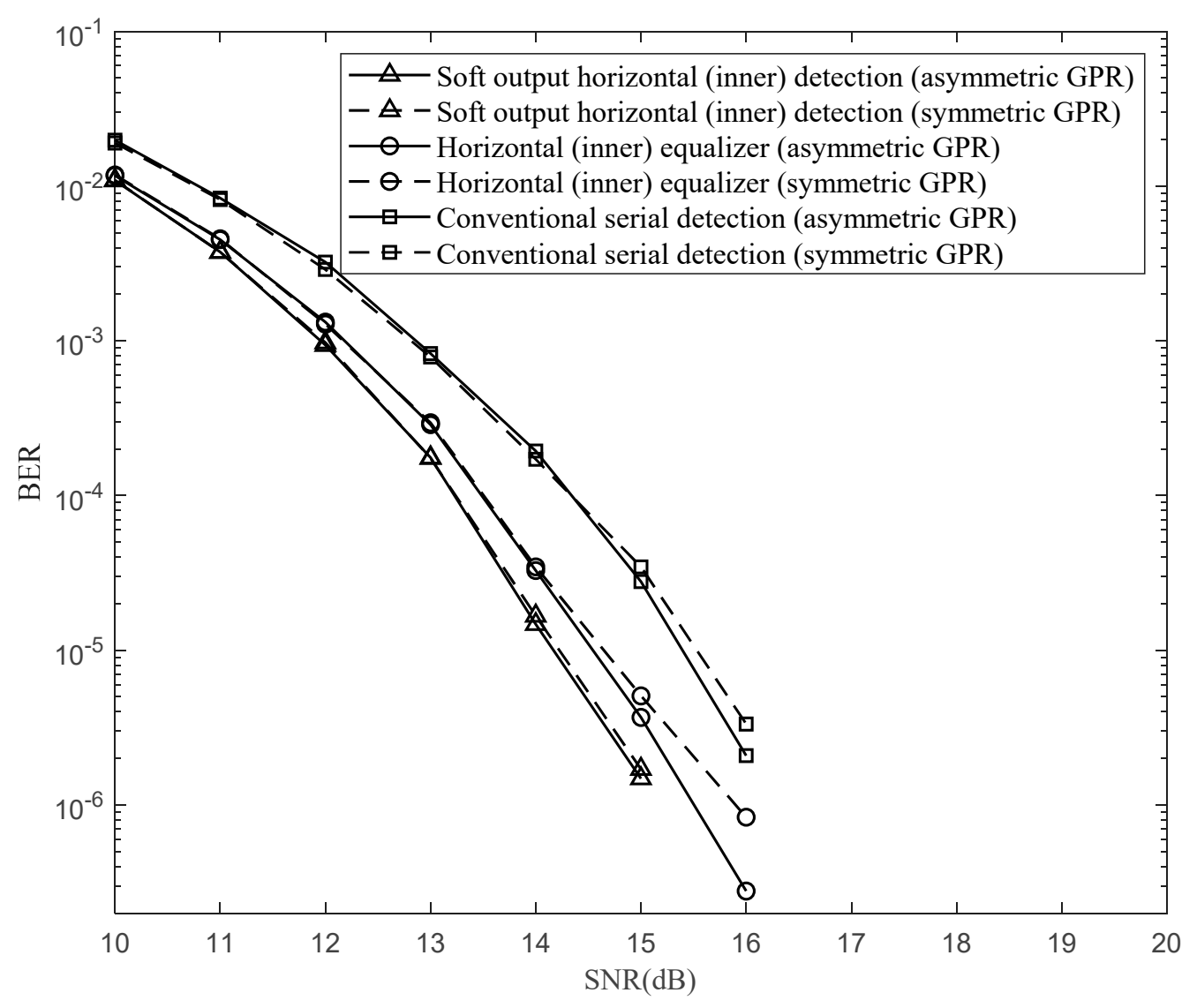

Figure 5. BER performance of the proposed models with 10\% TMR.

Meanwhile, the targets estimated for the channels with 10\% and 20\% TMR were

$$
\mathbf{G}_{10 \%}=\left[\begin{array}{ccc}
0.0428 & 0.4391 & 0.0428 \\
0.0974 & 1 & 0.0974 \\
0.0445 & 0.4567 & 0.0445
\end{array}\right]
$$


and

$$
\mathbf{G}_{20 \%}=\left[\begin{array}{ccc}
0.0288 & 0.4673 & 0.0288 \\
0.0617 & 1 & 0.0617 \\
0.0298 & 0.4833 & 0.0298
\end{array}\right],
$$

respectively. The target was calculated at $\mathrm{SNR}=20 \mathrm{~dB}$. Because of the effect of TMR, the channel coefficients were asymmetric. To estimate this asymmetry, an asymmetric GPR like (3) was applied to the BPMR systems. The results show that the asymmetric GPR target performs better than the symmetric GPR target when faced with TMR. In addition, we also experiment with the symmetric GPR like (1) by assigning the ITI coefficients to the average of $p_{1}$ and $p_{2}$.

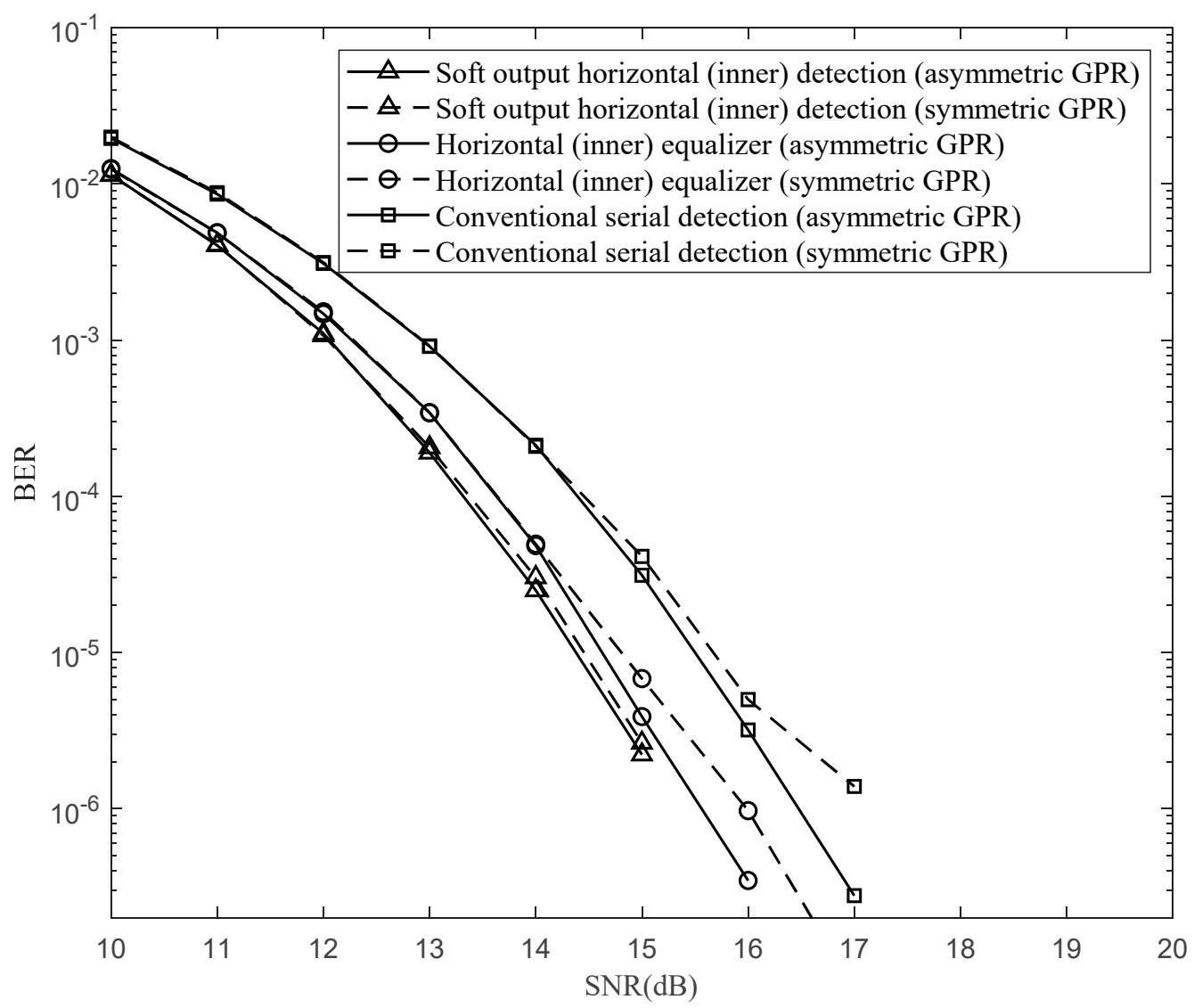

Figure 6. BER performance of the proposed models with $20 \%$ TMR, respectively.

Figure 7 shows the effect of TMR on serial detection with soft output for the inner detector. In fact, we do not know exactly what level TMR appears. Therefore, we simulated with SNR $=15 \mathrm{~dB}$ and TMR from $10 \%$ to $30 \%$. Two models proposed for the soft output for the inner detector outperformed the conventional serial detection model. However, the proposed models are more sensitive to TMR. This is because the soft output for the inner detector preserves interference information including information about TMR. Therefore, changes in TMR values also change the performance of the proposed model.

Finally, we simulated our proposed scheme with a $6 \%$ fluctuation in the position [17]. In this case, the coefficients are changed according to the position of the bit island. The results in Figure 8 show that soft output for the inner detector still achieved a gain of $\sim 2 \mathrm{~dB}$ at a BER of $10^{-5}$. However, two methods of soft-output achieve almost the same result because position fluctuation causes the target coefficients to lose the formats given in (1) and (3). 


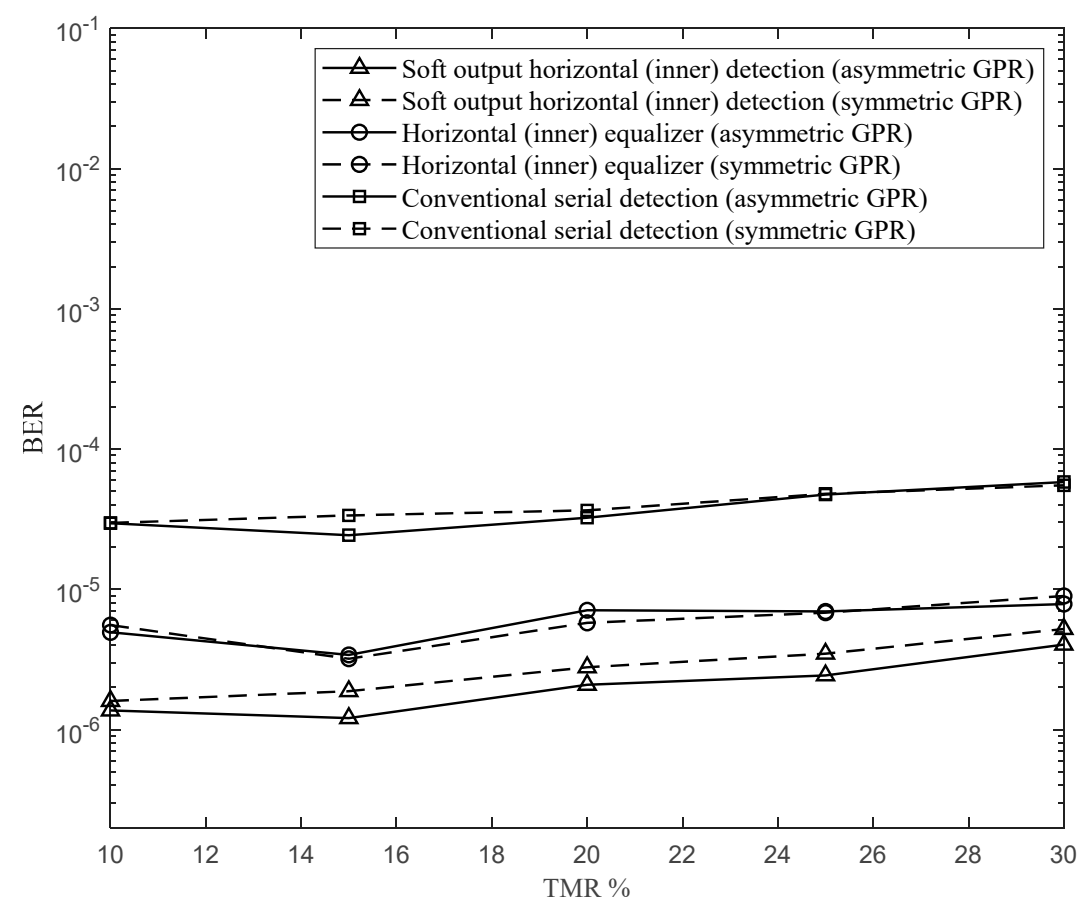

Figure 7. BER performance of the proposed models according to TMR.

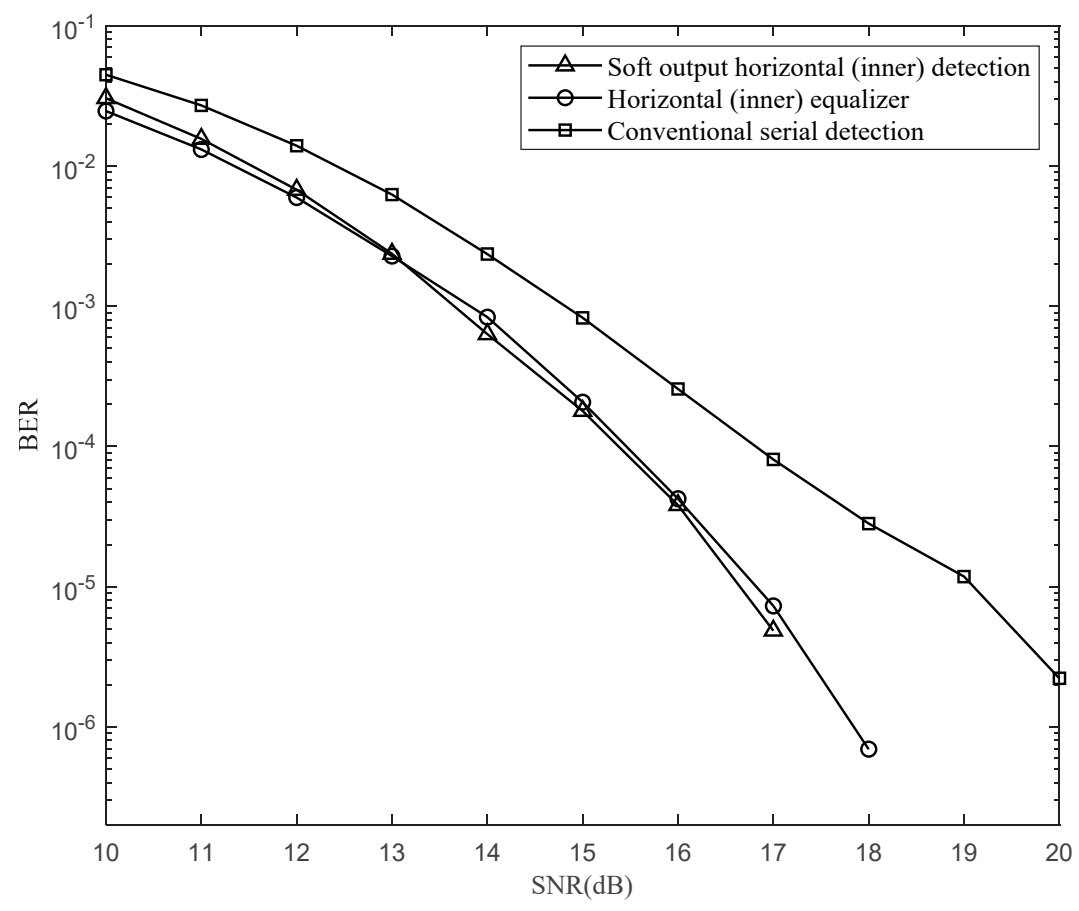

Figure 8. BER performance of the proposed models with $6 \%$ position fluctuation.

\section{Conclusions}

We have proposed soft output schemes for the inner detector in serial detection. Based on an equalizer and the Viterbi algorithm, our proposed model shows that the inner detector with soft-output achieves better BER performance than that of the inner detector with the six-level signal. In particular, the proposed model also brings high performance to channels with TMR. 
Author Contributions: Conceptualization, T.A.N. and J.L.; methodology, T.A.N. and J.L.; software, T.A.N.; validation, T.A.N. and J.L.; formal analysis, T.A.N.; investigation, T.A.N. and J.L.; writing-original draft preparation, T.A.N.; writing-review and editing, T.A.N. and J.L.; supervision, J.L.; project administration, J.L.; funding acquisition, J.L. All authors have read and agreed to the published version of the manuscript.

Funding: This work was supported by a National Research Foundation of Korea (NRF) grant funded by the Korea government (MSIT) (No. NRF-2019R1FA1046899).

Conflicts of Interest: The authors declare no conflict of interest.

\section{References}

1. Shiroishi, Y.; Fukuda, K.; Tagawa, I.; Iwasaki, H.; Takenoiri, S.; Tanaka, H.; Mutoh, H.; Yoshikawa, N. Future option for hdd storage. IEEE Trans. Magn. 2009, 45, 3816-3822. [CrossRef]

2. Zhu, J.G.; Lin, Z.; Guan, L.; Messner, W. Recording, noise, and servo characteristics of patterned thin film media. IEEE Trans. Magn. 2000, 36, 23-29. [CrossRef]

3. Chang, W.; Cruz, J.R. Inter-track interference mitigation for bit-patterned magnetic recording. IEEE Trans. Magn. 2010, 46, 3899-3908. [CrossRef]

4. Nguyen, T.A.; Lee, J. Error-correcting 5/6 modulation code for staggered bit-patterned media recording systems. IEEE Magn. Lett. 2019, 10,1-5. [CrossRef]

5. Buajong, C.; Warisarn, C. Improvement in bit error rate with a combination of a rate-3/4 modulation code and intertrack interference subtraction for array-reader-based magnetic recording. IEEE Magn. Lett. 2019, 10, 1-5. [CrossRef]

6. Kanjanakunchorn, C.; Warisarn, C. Soft-decision output encoding/decoding algorithms of a rate-5/6 citi code in bit-patterned magnetic recording (BPMR) systems. In Proceedings of the 34th International Technical Conference on Circuits/Systems, Computers and Communications, JeJu, Korea, 23-26 June 2019; pp. 1-4. [CrossRef]

7. Nabavi, S.; Kumar, B.V.K.V. Two-dimensional generalized partial response equalizer for bit-patterned media. In Proceedings of the IEEE International Conference on Communications, Glasgow, UK, $24-28$ June 2007; pp. 6249-6254. [CrossRef]

8. Nabavi, S.; Kumar, B.V.K.V.; Zhu, J. Modifying viterbi algorithm to mitigate intertrack interference in bit-patterned media. IEEE Trans. Magn. 2007, 43, 2274-2276. [CrossRef]

9. Wang, Y.; Kumar, B.V.K.V. Improved multitrack detection with hybrid 2-D equalizer and modified viterbi detector. IEEE Trans. Magn. 2017, 53, 1-10. [CrossRef]

10. Kim, J.; Lee, J. Partial response maximum likelihood detections using two-dimensional soft output viterbi algorithm with two-dimensional equalizer for holographic data storage. Jpn. J. Appl. Phys. 2009, 48, 03 A003. [CrossRef]

11. Kim, J.; Moon, Y.; Lee, J. Iterative two-dimensional soft output viterbi algorithm for patterned media. IEEE Trans. Magn. 2011, 47, 594-597. [CrossRef]

12. Jeong, S.; Kim, J.; Lee, J. Performance of bit-patterned media recording according to island patterns. IEEE Trans. Magn. 2018, 54, 1-4. [CrossRef]

13. Nguyen, T.A.; Lee, J. One-dimensional serial detection using new two-dimensional partial response target modeling for bit-patterned media recording. IEEE Magn. Lett. 2020, 11, 1-5. [CrossRef]

14. Nutter, P.W.; Ntokas, I.T.; Middleton, B.K.; Wilton, D.T. Effect of island distribution on error rate performance in patterned media. IEEE Trans. Magn. 2005, 41, 3214-3216. [CrossRef]

15. Nutter, P.W.; Ntokas, I.T.; Middleton, B.K.; Wilton, D.T. Tracking issues in high-density patterned media storage. In Proceedings of the IEEE International Magnetics Conference, Nagoya, Japan, 4-8 April 2005; pp. 1377-1378. [CrossRef]

16. Warisarn, C.; Arrayangkool, A.; Kovintavewat, P. An ITI-mitigating 5/6 modulation code for bit-patterned media recording. IEICE Trans. Electron. 2015, E98-C, 528-533. [CrossRef]

17. Nabavi, S.; Kumar, B.V.K.V.; Bain, J.A. Two-dimensional pulse response and media noise modeling for bit-patterned media. IEEE Trans. Magn. 2008, 44, 3789-3792. [CrossRef]

(C) 2020 by the authors. Licensee MDPI, Basel, Switzerland. This article is an open access article distributed under the terms and conditions of the Creative Commons Attribution (CC BY) license (http://creativecommons.org/licenses/by/4.0/). 